\title{
A Semantic Policy Sharing Infrastructure for Pervasive Communities
}

\author{
Vikash Kumar \\ The Telecommunications Research Center Vienna (FTW), Austria \\ kumar@ftw.at
}

\begin{abstract}
We propose a policy sharing infrastructure that enables a semantic rule created in one set of environmental, physical and contextual settings to be translated, while maintaining the underlying semantics, for use in a situation when those settings/parameters change. With an aim to enable sharing of useful intelligence described in the form of policies, we base our discussions on smart home and mobile commerce use cases.
\end{abstract}

Keywords: Policy sharing, Semantic Rules, Smart homes, mCommerce.

\section{Introduction and Motivation}

Rules are increasingly being used in semantic applications as well as in traditional IT systems to provide a formal and powerful way of representing information like individual preferences, privacy constraints etc 122 . To make such systems more versatile, there have been many initiatives that aim to transform a rule created in one semantic standard to one created in another 35 10. However, in all these cases there is no provision for a rule or a policy to be shared or reused among applications where its semantic meaning is preserved even while the context and other parameters of respective application environments change. This thesis will elaborate on a solution for an intelligent semantic policy sharing infrastructure that enables the agents of an application across different settings, context, environments, etc. to share and reuse semantic policies amongst themselves. For this we identify variables that affect the definition of a policy at different usage sites of the application and provide them as input to an underlying translation engine. The investigations are carried out in the use case of a smart home community as well as in an mobile commerce (m-commerce) setting.

Smart meters are increasingly being installed in common households in most countries and at the same time there is an increasing tendency of people residing in living communities like in apartments of a common large building in the current urban landscape. A natural next step in such a scenario would be to further utilize the advantages of Information Communication Technologies (ICT) in creating connected urban environments which promote increased partnerships among residents of a living community through better information sharing and transparency 11/2]. A part of such information sharing and exchange of ideas

\footnotetext{
1 A policy consists of one or more rules. 
can be in the form of policies that the resident of an apartment creates for energy saving and/or better user experience in his/her respective home.

In the m-commerce use case, we aim to use telecommunications (telco) specific information like identity, location, etc. in providing personalized advertisements (ads) to users based on their own preferences set in the form of policies. The proposed infrastructure would enable them in getting better recommendations by sharing efficient policies among acquaintances in a Web 3.0 environment.

The main research question to be investigated in this thesis is: For the same application, can a semantic policy created for one set of physical, environmental and contextual conditions and settings be effectively translated and applied into another different set of settings while preserving its core idea? Along the way, this work will also show how to translate the benefits achieved (in terms of cost/energy savings, etc.) by application of a policy from one environment to another.

\section{Related Work}

There has been considerable interest in the areas of rule interchange and profile matching in the semantic web community. RuleML 6] (Rule Markup Language) was the first initiative aimed at creating a unifying family of XML-serialized rule languages that includes all the web rules. The REWERSE I1 Rule Markup Language(R2ML) furthered this cause by proposing a comprehensive XML rule format by integrating languages like OCL, SWRL and RuleML 10.

W3C launched the Rule Interchange Format(RIF) [5] working group in 2005 tasked with producing a core rule language using which rules can be represented across all systems. The RIF framework for rule-based languages consists of a set of dialects which formally describes information about the syntax, semantics and XML serialization of a language. A semantics-enabled layered policy architecture has been proposed in [3] as an extension of W3C's Semantic Web architecture aimed at facilitating the exchange and management of policies created in multiple languages across the web.

Several projects have tried to utilize the benefits of smart meters and build applications and services around data collected by them 91112. Several others have suggested the use of mobile specific enablers for providing privacy aware services based on semantic rules for mobile users [13 14.

While this thesis takes inspirations from the existing works in the direction of rule interchange and sharing, the unique feature of the proposed infrastructure will be the translation of a policy created in one set of conditions into that in another set of conditions for the "same" application in a privacy aware manner. Therefore the focus won't be on application or language independence as proposed in other approaches.

\section{Approach and Methodology}

In order to re-use the ideas and best practices of different users, the first requirement would be to serialize their preferences and policies in a common format 


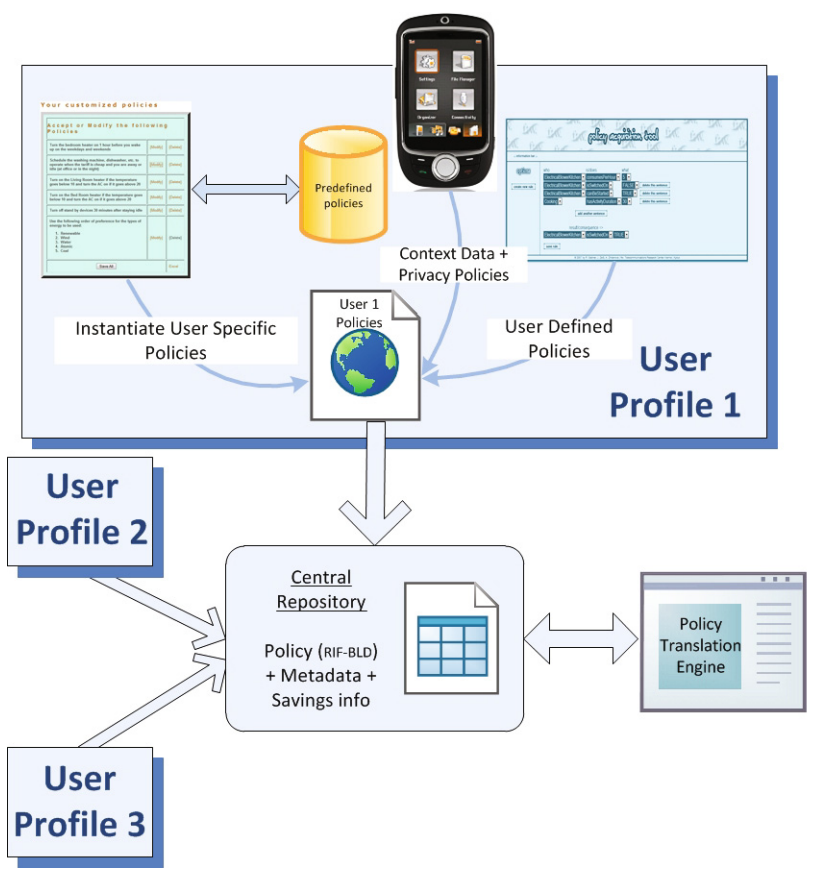

Fig. 1. The policy sharing infrastructure

(possibly RIF-BLD [5]) which could easily be adapted from one array of settings to another. Thereafter, for the translation, we need to first identify the static and dynamic parameters of a rule and then replace the dynamic ones with those matching the new set of conditions. For example, user $X$ 's policy: "Send me all offers for iPad3 from Saturn electronic store" will be translated for user $Y$ as "Send me all offers for Samsung Galaxy tab from Amazon" where $Y$ is interested in electronic ads but his/her profile shows an inclination for Samsung products (rather than Apple) and the shopping behavior shows transactions mostly from Amazon (rather than Saturn). The italicized and underlined parts represent the static and dynamic contents of the rule respectively. Other inferences affecting this translation obtained by reasoning over user profile and domain ontology, their online behavior and system policies may be facts like iPad3 is a device in Tablet category while Saturn is a shop in the category Electronic Store. A suitable technique for such a matching of ontology concepts needs to be developed. Another ontology containing the meta-policy information and other rules governing policy translation would be a part of this infrastructure. A central repository (Figure 1) will collect all the user policies annotated with their respective metadata containing information about their static and dynamic parameters, the perceived quantifiable advantage(s) achieved by their application, etc. It will also contain user and environment data like profile, preferences, temperature, etc. that will substitute the dynamic parameters of a rule. 
A Policy Translation Engine performs the actual translation of individual rules in a policy from one setting to another using data available in the repository. It also computes the perceived savings attained by the application of the policy in both the settings and recommends the translated policy to interested users. The overall research is planned in various phases described below.

Preliminary Study \& Analysis. A study of state of the art in various aspects of this project will be taken up. This would include a survey of existing rule based service enablers, semantic rule interchange standards, utilizing data from smart homes and personalized recommendation based m-commerce initiatives.

Specification of the Infrastructure. Based on the above inputs, a specification of the proposed semantic policy sharing infrastructure will be laid out including formal definitions of individual components.

Prototype Implementation. The prototype implementation would be done in several stages comprising of development of each module of the system followed by an integration phase. The modules envisaged as of now are (i) policy creation \& modification tool, (ii) ontology development, (iii) the underlying policy translation module, (iv) intelligent context manager and recommendation engine for adaptation in telco scenario and (v) integration.

Prototype Evaluation. A separate user evaluation will be conducted for each module individually and for the prototype as a whole in context of both the use cases based on various parameters like accuracy, user acceptance, etc.

\section{Preliminary Results}

At the end of this thesis, we expect to have a working prototype of the policy translation infrastructure in the mentioned use cases. The focus of smart home use case would be to allow sharing of effective energy saving policies in a resident community and the evaluations would investigate the semantic similarity of the translated policy along with preciseness of calculated savings data. The m-commerce use case will primarily aim at using sharable policies as a tool for preserving user privacy while still being able to infer useful information from their publicly shared data for ad recommendations. Evaluations will be based on relevance of ads served by effect of original and translated policies.

\section{Conclusions}

In this paper, we introduced the idea of a semantic policy translation infrastructure and described some related work which would form a starting point of the research carried out in this thesis. Thereafter, we also mentioned some preliminary results of the work so far. According to the methodology shown in Section 3 , the next major steps are to complete the policy translation engine, context management system as well as the policy recommendation tool. Finally, we intend to test our hypothesis through extensive user tests of the infrastructure proposed in this paper. 
Acknowledgments. This work has been partially supported by FFG funded SESAME-S project [4] and partly by APSINT [8]. The Telecommunications Research Center Vienna (FTW) is supported by the Austrian government and the City of Vienna within the competence center program COMET. Thanks to Dr. Anna Fensel and Prof. Gabriele Kotsis for supervising this work.

\section{References}

1. Toninelli, A., Jeffrey, B.M., Kagal, L., Montanari, R.: Rule-based and Ontologybased Policies: Toward a hybrid approach to control agents in pervasive environments. In: Semantic Web and Policy Workshop (2005)

2. Grosof, B.N.: Representing E-Business Rules for the Semantic Web: Situated Courteous Logic Programs in RuleML. In: Workshop on Information Technologies and Systems, WITS 2001 (2001)

3. Hu, Y.J., Boley, H.: SemPIF: A Semantic meta-Policy Interchange Format for Multiple Web Policies. In: Proc. of the 2010 IEEE/WIC/ACM International Conference on Web Intelligence and Intelligent Agent Technology (WI-IAT 2010), vol. 1, pp. 302-307. IEEE Computer Society, Washington, DC (2010)

4. The SESAME-S Project, http://sesame-s.ftw.at/

5. Rule Interchange Form, http://www.w3.org/2005/rules/

6. RuleML, http://www.ruleml.org/

7. Zhdanova, A.V., Zeiß, J., Dantcheva, A., Gabner, R., Bessler, S.: A Semantic Policy Management Environment for End-Users and Its Empirical Study. In: Schaffert, S., Tochtermann, K., Auer, S., Pellegrini, T. (eds.) Networked Knowledge - Networked Media. SCI, vol. 221, pp. 249-267. Springer, Heidelberg (2009)

8. The APSINT Project, http://www.apsint.ftw.at/

9. Kumar, V., Tomic, S., Pellegrini, T., Fensel, A., Mayrhofer, R.: User Created Machine-Readable Policies for Energy Efficiency in Smart Homes. In: Proc. of the Ubiquitous Computing for Sustainable Energy (UCSE 2010) Workshop at the 12th ACM International Conference on Ubiquitous Computing, UbiComp 2010 (2010)

10. Bădică, C., Giurca, A., Wagner, G.: Using Rules and R2ML for Modeling Negotiation Mechanisms in E-Commerce Agent Systems. In: Draheim, D., Weber, G. (eds.) TEAA 2006. LNCS, vol. 4473, pp. 84-99. Springer, Heidelberg (2007)

11. Möller, S., Krebber, J., Raake, E., Smeele, P., Rajman, M., Melichar, M., Pallotta, V., Tsakou, G., Kladis, B., Vovos, A., Hoonhout, J., Schuchardt, D., Fakotakis, N., Ganchev, T., Potamitis, I.: INSPIRE: Evaluation of a Smart-Home System for Infotainment Management and Device Control. In: Proc. of the LREC 2004 International Conference, Lisbon, Portugal, pp. 1603-1606 (2004)

12. Kamilaris, A., Trifa, V., Pitsillides, A.: HomeWeb: An application framework for Web-based smart homes. In: 18th International Conference on Telecommunications (ICT), pp. 134-139 (2011)

13. Gandon, F.L., Sadeh, N.M.: Semantic web technologies to reconcile privacy and context awareness. Journal of Web Semantics 1, 241-260 (2004)

14. Toninelli, A., Montanari, R., Kagal, L., Lassila, O.: A Semantic Context-Aware Access Control Framework for Secure Collaborations in Pervasive Computing Environments. In: Cruz, I., Decker, S., Allemang, D., Preist, C., Schwabe, D., Mika, P., Uschold, M., Aroyo, L.M. (eds.) ISWC 2006. LNCS, vol. 4273, pp. 473-486. Springer, Heidelberg (2006) 